\title{
Nuevas perspectivas terapéuticas en tuberculosis
}

\author{
CARLOS PEÑA M.* y VICTORINO FARGA C.*
}

\section{New perspectives in tuberculosis treatment}

To advance in the control and elimination of tuberculosis (TB) we must achieve a high level of effectiveness in the prevention of TB in populations infected by Mycobacterium tuberculosis. Latent TB prevention success with current therapies (single isoniazid or in combination with rifampicin) is close to $60 \%$. We also must offer a high level of treatment success in first-line drugs sensitive TB patients. With currently available drugs (isoniazid, rifampicin, ethambutol and pyrazinamide) treatment success should reach at least $95 \%$. Drug side reactions together with the lengthen treatment of infection and disease (6 months) decrease the compliance to these therapies. In Multi-Drug-Resistant TB (MDR-TB), therapies are even longer (20 months according to WHO's recommendation) and much less tolerated, with rates of success under 50\%. New trials for latent TB using rifapentin and isoniacid; combined fixed-dose of first-line drugs in sensible TB, and the addition of new drugs (fluorquinolones, bedaquiline, delamanid and linezolid) in multi-drug resistant TB, together with shorter regimens of 12 months duration which include Clofazimine (experience in Cameroon with modification of a 9 months trial previously used in Bangladesh showing $89 \%$ cure) are discussed in this article.

Key words: Tuberculosis treatment, Tuberculosis latent infection, new tuberculosis drugs, MultiDrug-Resistant-Tuberculosis (MDR-TB).

\section{Resumen}

Para el control y eliminación de la tuberculosis se debe lograr un alto grado de eficacia en la prevención del desarrollo de tuberculosis en la población infectada por Mycobacterium tuberculosis. Esta prevención, con las terapias actuales de la tuberculosis latente (isoniazida sola o combinada con rifampicina), es cercana al 60\%. También debemos alcanzar una alta tasa de curación para los enfermos con tuberculosis sensible a los fármacos de primera línea (vírgenes a tratamiento). Con los fármacos actualmente disponibles (isoniazida, rifampicina, etambutol y pirazinamida) esta curación debería alcanzar a no menos del 95\%. La regular tolerancia y reacciones colaterales de los fármacos y el largo tiempo que demandan las terapias de la infección y de la enfermedad (6 meses) atenta contra su adherencia. En el caso de la Tuberculosis Multi-Drogo-Resistente (TB-MDR), los tratamientos son aún más prolongados (20 meses según recomienda la OMS actualmente) y menos tolerados, siendo sus tasas de curación inferiores a 50\%. Se analizan nuevos esquemas para el tratamiento de la tuberculosis latente usando rifapentina asociada a isoniacida; dosis fijas combinadas de fármacos de primera línea para tuberculosis sensibles, y asociación de fármacos antiguos y nuevos (fluoroquinolonas, bedaquilina, delamanid y linezolid) para el tratamiento de las tuberculosis multirresistentes. También se presentan nuevos esquemas acortados, de 12 meses de duración, que incluyen clofazimina (experiencia en Camerún con modificación del esquema de 9 meses usado previamente en Bangladesh, con tasas de curación de 89\%).

Palabras clave: Tratamiento de la tuberculosis, tuberculosis latente, nuevas drogas antituberculosas, Tuberculosis Multi-Drogo-Resistente (TB-MDR).

* Comité Técnico Programa de Control y Eliminación de la Tuberculosis, Ministerio de Salud de Chile. 


\section{Introducción}

Después de largos años de sequía farmacológica, durante el último decenio se han ido introduciendo una serie de fármacos nuevos contra la tuberculosis (TB), tanto en su etapa de enfermedad, como para prevenir las reactivaciones en su etapa latente. Y, lo que es más importante, se están ensayando nuevas combinaciones de drogas antituberculosas que tienen ventajas frente a los esquemas actuales y prometen cambiar los enfoques terapéuticos de los últimos años. Tres son las principales promesas: 1) Descubrimiento de nuevos fármacos con novedosas propiedades antituberculosas, con reevaluación de medicamentos ya conocidos; 2) Estudio de nuevas asociaciones terapéuticas que permitan abreviar y simplificar el manejo de las TB-MDR (tuberculosis multidrogo-resistentes) y 3) Intentos de potenciar los esquemas primarios con miras a abreviar el tratamiento de las tuberculosis sensibles.

La prevención de la reactivación de la tuberculosis en infectados por Mycobacterium tuberculosis (tuberculosis latente) mediante la administración de fármacos ha mostrado ser efectiva, logrando hasta un $60 \%$ de reducción del riesgo de evolucionar a enfermedad con el uso de isoniazida $(\mathrm{H})$ administrada diariamente durante 6 a 9 meses $^{1} \mathrm{o}$ asociada a rifampicina (R) durante 3 meses $^{2}$. Sin embargo, estos resultados se ven afectados por la baja adherencia en su autoadministración. Por lo tanto, se necesita contar con nuevas terapias, más abreviadas y eficientes.

Respecto al tratamiento de los casos nuevos de tuberculosis, el esquema actual con la asociación de $\mathrm{H}, \mathrm{R}$, etambutol (E) y pirazinamida (Z) brinda más de un $95 \%$ de curación ${ }^{3-4}$. Sin embargo, la regular tolerancia y aceptabilidad de este esquema y su aún prolongada duración de 6 meses, son factores desfavorables que comprometen la adherencia al tratamiento. En Chile, la cohorte de enfermos nuevos tratados el año 2013 mostró una curación de sólo $79,7 \%$, con una pérdida de seguimiento (abandono más de 30 días) de un $6,8 \%$. Por otra parte, de los pacientes antes tratados ${ }^{5}$ sólo curó el $55,7 \%$, con $21 \%$ de pérdida de seguimiento. Sin duda se requieren nuevos esquemas, mejor tolerados y más breves, para el control de estos dos grupos, que en forma primaria o después de un primer tratamiento, pueden ser portadores de tuberculosis con distintos grados de multirresistencia a las drogas de primera línea.

En la Tuberculosis Multi-Drogo-Resistente (TB-MDR), resistente a isoniacida y rifampicina, los esquemas internacionales recomendados tienen débiles evidencias sobre su eficacia. Se basan en meta-análisis con $\mathrm{Z}$ asociada a otros 4 fármacos (incluyendo una fluoroquinolona y etionamida) y a una terapia antiviral precoz en los casos infectados con $\mathrm{VIH}^{6}$. En un metaanálisis, que incluyó 32 estudios observacionales con 9.153 pacientes, se detectó una baja eficacia (éxito de $54 \%$ ), alta letalidad (15\%) y pérdida de seguimiento $(23 \%)$, por deficiente tolerancia a terapias prolongadas, complejas y costosas ${ }^{7}$. Pero se destacó la mayor eficacia de las asociaciones con fluoroquinolonas. Los esquemas actualmente recomendados por la OMS tienen una duración de 20 meses, lo que atenta contra la adherencia a estos tratamientos.

En Chile la terapia para casos de TB-MDR tienen una duración mínima de 12 meses (habitualmente 18 meses dependiendo de la evolución bacteriológica). Utiliza moxifloxacino (Mox), kanamicina $(\mathrm{K})$, etionamida $(\mathrm{Et}), \mathrm{E}$ y Z. Los resultados en la cohorte de pacientes tratados el año 2012 señalaron una curación de $45 \%$, con $27 \%$ de pérdidas de seguimiento.

A continuación se detallan los resultados de nuevos esquemas de tratamiento para TB latente, TB sensible y TB-MDR.

\section{Nuevas terapias para la tuberculosis latente}

La rifapentina es una rifamicina de acción prolongada que actúa inhibiendo la RNA polimerasa bacteriana. Su uso asociada a $\mathrm{H}$ una vez por semana, durante 3 meses, muestra una eficacia similar al empleo de $\mathrm{H}$ sola durante 9 meses, pero la proporción de personas que según un estudio completan la terapia es mayor $(82,1$ versus $69 \%$, $\mathrm{p}<0,001)^{8}$. Sin embargo, debemos considerar que estos protocolos son administrados bajo supervisión directa y no auto-administrados como sucede en la terapia habitual de TB latente con $\mathrm{H}$, lo que confiere un sesgo de mayor adherencia. La toxicidad hepática de este nuevo esquema es baja $(0,3 \%$ versus $0,2 \%$ con $H, p<0,001)$ pero puede aparecer hipotensión y trombocitopenia. La R, al ser inductora del citocromo CYP3A reduce la concentración sanguínea de fármacos que usan esta vía metabólica. No se recomienda en personas con infección VIH, en menores de 2 años y en embarazadas o en lactancia.

\section{Nuevas terapias para la tuberculosis sensible}

\subsection{Dosis fijas combinadas (DFC)}

A pesar que esta estrategia no es reciente en el mundo, en nuestro país está actualmente 
disponible para su empleo como primera línea de tratamiento. Para la fase diaria cada tableta contiene H $75 \mathrm{mg}$, R $150 \mathrm{mg}$, E $275 \mathrm{mg} \mathrm{y} \mathrm{Z}$ $400 \mathrm{mg}$ y para la fase trisemanal H $150 \mathrm{mg}$ y R $150 \mathrm{mg}$. La dosificación habitual considera 4 tabletas como dosis unitaria en ambas fases de terapia. Aunque las DFC no han demostrado una eficacia mayor ni menos efectos colaterales que el uso de dosis separadas de los medicamentos, su tolerancia y aceptabilidad es mayor y su costo menor. Además, la asociación de las drogas en un comprimido previene el desarrollo de resistencia a fármacos cuando los tratamientos no son totalmente supervisados.

\subsection{Esquemas primarios más breves}

Las fluoroquinolonas (FQN) son potentes fármacos bactericidas que inhiben la girasa del ADN de las micobacterias, impidiendo su síntesis. En nuestro medio el más utilizado es el moxifloxacino (Mox) que se emplea preferentemente en las TB-MDR, aunque hay experiencias de su empleo en TB sensibles intentado abreviar la duración del tratamiento, como se analiza a continuación:

a) El ensayo Fase III denominado REMoxTB ${ }^{9}$ incluyó pacientes de varios continentes, excluyendo casos VIH con CD4 $<250$ céls $/ \mathrm{mm}^{3}$. En este estudio se utilizaron esquemas más abreviados, de 4 meses de duración, con asociaciones similares a las del tratamiento estándar de 6 meses con 4 drogas (HREZ), en los cuales el Mox sustituyó a la $\mathrm{H}$ en un grupo y al $\mathrm{E}$ en otro. Se midió la eficacia y el grado de conversión del cultivo, es decir el efecto bactericida de estos cambios, comparándolos con la terapia estándar administrada por 6 meses. El esquema en el cuál se sustituyó al E mostró una mayor y más precoz negativización de los cultivos de expectoración, pero con un menor grado de éxito terapéutico que el alcanzado con la terapia estándar $(85 \%$ versus $92 \%$ ). El grupo que sustituyó a $\mathrm{H}$ por Mox mostró el mismo poder de conversión bacteriológica de los cultivos que el esquema estándar, pero un éxito menor ( $80 \%$ versus $92 \%)$.

A pesar del bajo nivel de fracasos en ambos grupos $(<1 \%)$, la proporción de recaídas al sustituir a la $\mathrm{H}$ llegó a $12 \%$ y al sustituir al E alcanzó a $9 \%$, lo que está muy por sobre la proporción de recaídas del tratamiento estándar $(2,4 \%)$. En conclusión, este ensayo acortado a 4 meses con Mox fracasó en demostrar una eficacia no inferior con respecto a la terapia estándar de 6 meses de duración.

b) En otro ensayo, el estudio RIFAQUIN ${ }^{10}$ el Mox sustituyó a $\mathrm{H}$ en la fase diaria de 2 meses, seguido de una fase intermitente en que se asoció
Mox con rifapentina ( $\mathrm{Rp}$ ) en 2 grupos, uno de 4 y otro de 6 meses de duración. El grupo que continuó recibiendo sólo 2 meses más de Mox asociado a $\mathrm{Rp}$ en dosis de $900 \mathrm{mg}$, administrados bisemanalmente, tuvo una proporción de recaídas de $15,8 \%$ (versus $3 \%$ con el esquema estándar). El otro grupo que continuó recibiendo 4 meses de Mox asociado a Rp en dosis de $1.200 \mathrm{mg}$ una vez por semana, completando 6 meses de terapia, sólo tuvo $3,2 \%$ de recaídas.

El esquema que recibió Rp sólo 2 meses en la fase intermitente, con un total de sólo 4 meses de tratamiento, tuvo una respuesta desfavorable con una mezcla de recaídas, muertes y fracasos, cercana al $18 \%$, fracasando en demostrar una eficacia no inferior al tratamiento estándar. Por otra parte, el grupo tratado por 6 meses con Mox alcanzó una proporción de respuesta desfavorable similar al grupo control (3,2 versus 4,9\%). Debe señalarse que la conversión del cultivo a los 2 meses en los esquemas con Mox fueron similares al grupo control $(90,4$ versus $85,3 \%)$.

Actualmente las FQN se usan en TB sensible sólo como alternativa a la toxicidad o resistencia a algún fármaco de primera línea. Por el momento no han demostrado que puedan acortar los tratamientos actuales. Son medicamentos relativamente seguros (la tendinitis y el daño hepático son poco frecuentes), pero deben evitarse en personas con intervalo QT prolongado al electrocardiograma, en cardiópatas o los que estén recibiendo antiarrítmicos.

\section{Nuevos fármacos y nuevos esquemas para las tuberculosis multirresistentes}

Los tratamientos actuales de la TB-MDR y de las Tuberculosis Extremadamente Resistentes (TB-XDR), que además son resistentes a las quinolonas e inyectables como la kanamicina, propiciados por la OMS, utiliza combinaciones de fármacos de segunda línea y prolongados tratamientos, con alta proporción de efectos colaterales que influyen en la menor adherencia de los pacientes y su reducida eficacia, como ya se ha señalado. Por estas razones, se están ensayando nuevos medicamentos y esquemas más acortados de tratamiento para las TB-MDR.

\section{Bedaquilina (BDQ)}

Este fármaco es una diarylquinolina conocida como TMC207, que actúa inhibiendo la ATP sintetasa de la membrana bacteriana. Se metaboliza por el citocromo P450 y CYP3A4. Los ensayos terapéuticos de fase II comparando la adición de 
BDQ contra placebo ${ }^{11}$ en los primeros 6 meses de esquemas de sólo 12 a 18 meses de duración que contienen FQN, E, Et, Z y Cicloserina mostraron una más rápida negativización de la baciloscopia (83 versus 125 días en el grupo control) y una mayor conversión del cultivo (79 versus $58 \%$ ) a las 24 semanas en que culminó su uso. Además, se constató un mayor éxito terapéutico a los 30 meses (58\% versus $32 \%)$ cuando se empleó $\mathrm{BDQ}$, aunque se informó un exceso de muertes relacionadas con el fármaco (13 versus $2 \%$ con el grupo placebo). Se requieren mayores estudios confirmatorios, ya que hay un meta-análisis ${ }^{7}$ que muestra un $15 \%$ de mortalidad para la TB-MDR con los esquemas actuales. Este ensayo incluyó un $62 \%$ de casos resistentes a $\mathrm{Z}$ y un $83 \%$ con enfermedad cavitaria, los cuales son factores adversos al éxito terapéutico.

Entre los efectos secundarios de la BDQ figuran las náuseas (38\%), artralgias (32,9\%), cefaleas $(27,8 \%)$, hemoptisis $(17,7 \%)$, elevación de transaminasas $(8,9 \%)$ y prolongación del intervalo QT (hasta 26,6\%). El nombre comercial de este fármaco es Sirturo ${ }^{\circledR}$ y está preparado en tabletas de $100 \mathrm{mg}$. La dosis a usar es de $400 \mathrm{mg}$ diarios durante 1 a 2 semanas, seguidos de la misma dosis, pero tres veces por semana durante 22 semanas.

\section{Delamanid (DLM)}

Este fármaco es un nitroimidazol conocido como OPC-67683. Inhibe la síntesis del ácido micólico, un constituyente de la pared de las micobacterias. Existen evidencias que demuestran que el uso de DLM en dosis de 200 o 400 mg/día tiene efectos similares (alcanzan la misma biodisponibilidad para una máxima acción bactericida) y que lo importante es el tiempo de tratamiento. En un estudio multinacional de 9 países con 481 pacientes, efectuado entre el 2008 y 2010 se constató que con la administración de DLM durante 2 meses (trial NCT00685360) ${ }^{12}$ se logró la conversión del cultivo hasta en $45,4 \%$ de los casos, versus $29,6 \%$ en el grupo sin DLM. Este estudio incluyó algunos pacientes VIH, pero sólo si tenían CD4 $>350$ céls $/ \mathrm{mm}^{3}$. Se excluyeron los enfermos con intervalo QT prolongado ( $>450 \mathrm{~ms}$ en hombres y $>470 \mathrm{~ms}$ en mujeres).

En otra investigación con seguimiento de los pacientes durante 24 meses (trial 242-07-204 y 242-07-208 $)^{13}$ se señaló una reducción de la mortalidad a $<1 \%$ al utilizar DLM durante 6 meses, versus $8,3 \%$ en el grupo de control con placebo o con su uso por menos de 2 meses, con un éxito terapéutico del $74,5 \%$ versus $55 \%$ en el grupo con placebo o con menos de 2 meses de DLM.
Este estudio incluyó casos de TB-XDR, los que con el empleo de DLM por 6 meses tuvieron un éxito de $61,4 \%$ y no se registraron muertes, en tanto que con menos de 2 meses de DLM la mortalidad fue de $25 \%$.

Los efectos adversos del DLM son de frecuencia similar entre los 200 y $400 \mathrm{mg} /$ día. En orden de frecuencia: náuseas $(38,3 \%)$, vómitos $(33 \%)$, mareos $(30,2 \%)$, insomnio $(28 \%)$, cefalea $(24 \%)$, dolor abdominal (24\%), hipokalemia (15\%), hiperuricemia $(12 \%)$, tinnitus $(11 \%)$, parestesias $(11 \%)$, temblor $(11 \%)$ y anemia $(7 \%)$. La prolongación del intervalo QT se relaciona con la dosis (al usar $100 \mathrm{mg} \mathrm{c} / 12 \mathrm{~h}$ aparece en el 10\% y con $200 \mathrm{mg} \mathrm{c} / 12 \mathrm{~h}$ en el 13\%). También dependen de los niveles de albúmina (no se debe utilizar DLM con niveles $<2,8 \mathrm{~g} / \mathrm{dl}$ ). Si el intervalo QT se prolonga más de $500 \mathrm{~ms}$ debe suspenderse el fármaco. Debemos considerar además su interacción con otros fármacos inductores de CYP3A4. La carbamazepina, fenitoína y rifampicina reducen los niveles de DLM y deben evitarse, mientras que los fármacos inhibidores de este citocromo, como claritromicina, azoles, lopinavir y ritonavir, aumentan la concentración de metabolitos del DLM, los que prolongan el intervalo QT. El nombre comercial del DLM es Deltyba ${ }^{\circledR}$ y está preparado en tabletas de $50 \mathrm{mg}$. La dosis recomendada es de $100 \mathrm{mg} \mathrm{c} / 12 \mathrm{~h}$ durante 24 semanas.

\section{Nuevas terapias acortadas para tuberculosis sensibles y resistentes}

Se trata de estudios con diferentes combinaciones de drogas de primera, segunda y tercera línea que permitirían tratar tanto casos nuevos de tuberculosis, como pacientes antes tratados y resistentes a drogas, con esquemas más abreviados que los actuales. Serían más aplicables en países de alta endemia de tuberculosis, con elevada proporción de multirresistentes en la población.

a) Experiencia de Bangladesh ${ }^{14}$ con esquemas de 9 meses. Las primeras evidencias surgieron con uno de los ensayos efectuados en 206 pacientes reclutados entre 1997 y 2007 y corresponde al esquema de 4 meses diarios que reúne $\mathrm{K}$, clofazimina, gatifloxacino, $\mathrm{E}, \mathrm{Z}$ y protionamida, seguidos de 5 meses diarios con gatifloxacino, E, Z y clofazimina. Este esquema alcanzó un éxito de $87,8 \%$, con $5,4 \%$ de muertes, $5,8 \%$ de abandonos y sólo $0,5 \%$ de recaídas. Posteriormente, en la cohorte reclutada entre el 2005 y 2011 con 515 pacientes ${ }^{15}$, el mismo esquema produjo un éxito de $84,5 \%$, pero sólo la mitad de los enfermos completó la terapia en 9 meses; 
95\% demoró 12 meses en completarla debido a la demora en la conversión del cultivo y a la presencia de enfermedad pulmonar extendida. De los pacientes que concluyeron con éxito el tratamiento el $82,3 \%$ no presentó recaídas en el seguimiento a 2 años. Las muertes alcanzaron sólo a 5,6\% y la mitad de ellas se presentaron dentro de los primeros 60 días de terapia. Los abandonos fueron $7,8 \%$ y también la mitad de ellos ocurrió en los primeros 60 días. Las reacciones colaterales a fármacos más frecuentes fueron los vómitos $(21,6 \%)$. Este esquema logró negativizar el cultivo a los 2 meses en el $93 \%$ de los casos con la asociación de gatifloxacino en altas dosis, una quinolona de tercera generación, porque aún no se ha demostrado un nivel de tolerancia seguro con el empleo de altas dosis de Mox o levofloxacino.

Actualmente está en marcha un protocolo (ISRCTN78372190 llamado STREAM) ${ }^{16}$ de la Unión Internacional contra la Tuberculosis y Enfermedades Respiratorias (UICTER) para corroborar estos estudios y evaluar la eficacia y seguridad de dosis de 600 a $800 \mathrm{mg}$ de Mox.

b) Experiencia en Camerún ${ }^{17}$ auspiciada por la UICTER. Esta terapia de 12 meses de duración utiliza dosis menores de gatifloxacino $(400 \mathrm{mg}$ diarios) asociado a clofazimina $100 \mathrm{mg}$, protionamida $(15-20 \mathrm{mg} / \mathrm{kg}), \mathrm{E}(25 \mathrm{mg} / \mathrm{kg})$ y Z (30-40 $\mathrm{mg} / \mathrm{kg}$ ), con un refuerzo inicial de 4 meses de $\mathrm{K}$ $(15-20 \mathrm{mg} / \mathrm{kg})$, junto a H $(5 \mathrm{mg} / \mathrm{kg})$. (Se agregó $\mathrm{H}$ porque en la cohorte previa de Bangladesh el esquema que utilizó $\mathrm{H}$ obtuvo mejores resultados). Esta asociación, empleada principalmente en pacientes TB-MDR y TB-XDR, con fracasos previos de varios tratamientos antituberculosos, alcanzó un éxito de 89,3\% y no presentó recaídas a 2 años de seguimiento. La conversión del cultivo se obtuvo en el 99,2\% al final del $3^{\text {er }}$ mes de terapia. Como reacciones colaterales se presentaron náuseas y vómitos, por la protionamida, durante las primeras semanas. El hipotiroidismo (protionamida), neuritis óptica (E), alteraciones de la glicemia (gatifloxacino), insuficiencia renal $(\mathrm{K})$ y hepatitis $(\mathrm{Z})$ fueron muy excepcionales.

La eficacia de la terapia de TB-MDR es mayor cuando existe sensibilidad a la $\mathrm{Z}$ y si hay baja probabilidad de resistencia a los fármacos de $2^{\mathrm{a}}$ línea en pacientes sin infección por VIH. Las terapias de las TB-XDR son más pobres, pero ofrecen mejores resultados cuando los esquemas se asocian con clofazimina $\mathrm{y}^{18}$. El uso de altas dosis de $\mathrm{H}$ no está claramente demostrado que sea necesario y sólo se ha utilizado en el $8 \%$ de los esquemas evaluados en los meta-análisis ${ }^{19}$.

\section{Antiguos fármacos resucitados}

\section{Linezolid (LZD)}

En caso de resistencia a las drogas de $1^{\mathrm{a}}$ y $2^{\mathrm{a}}$ línea o para intentar acortar la duración de la terapia, puede recurrirse al uso de BDQ, DLM y linezolid (LZD). El LZD es una oxazolididona y como fármaco bactericida actúa inhibiendo la síntesis proteica al ligarse al ribosoma bacteriano (impide la formación del complejo ribosomal 70S). Tiene interacciones con numerosos fármacos (ciclobenzaprinas, levodopa, meperidina, sibutramina, pseudoefedrina, bupropión, paroxetina, trazodona y fluoxetina) con los que no debe administrarse. Además, si se están recibiendo inhibidores de la monoaminoxidasa, la ingesta de alimentos ricos en tiramina puede ocasionar hipertensión. La evaluación realizada en Shangai $^{20}$ en 18 pacientes no VIH (15 eran TB-XDR) con fracaso a terapia de TB-MDR (tratados por 39 meses previamente con diversos medicamentos), que usaron LZD entre el 2007 y 2010 por un período promedio de 6 meses, la mayoría con dosis de $1.200 \mathrm{mg}$ diarios por vía intravenosa, asociados a 4-8 fármacos contra TB-MDR, mostró un $50 \%$ de éxito. Pero un $94 \%$ de los pacientes presentaron una reacción adversa a los medicamentos: alteración hematológica en $72 \%$ y problemas gastrointestinales en $83 \%$. Entre las alteraciones hematológicas en el $67 \%$ fue anemia, 39\% leucopenia y $17 \%$ trombocitopenia. La neuropatía y el rash alérgico se presentaron en el 56\%. La mayoría de estas reacciones son reversibles al suspender LZD y tratar la reacción. Sólo un caso requirió suspender definitivamente el medicamento. Los autores destacaron la alta proporción de reacciones adversas. Aún no se conoce con exactitud cuál es la dosis óptima que mantenga una alta eficacia con mínima toxicidad.

Un meta-análisis de 12 estudios recolectando 121 pacientes de 11 países en 3 continentes ${ }^{21}$ informó que más del $90 \%$ de los casos tratados en diferentes esquemas que contenían LZD (promedio 300 días de uso) lograron la conversión bacteriológica en un promedio de 43,5 días para la baciloscopia y 61 días para el cultivo, independiente de la dosis y que el éxito alcanzó al 81,8\% tanto en pacientes que usaron menos de $600 \mathrm{mg}$, como más de $600 \mathrm{mg}$ diarios. Existieron muchas reacciones adversas $(58,9 \%)$ proporcionales a las dosis (más de $600 \mathrm{mg}$ : $74,5 \%$ y menos de $600 \mathrm{mg}: 46,7 \%$ ). La anemia se detectó en $38,1 \%$, neuropatía en $47,1 \%$, trombocitopenia en $11,8 \%$, neuritis óptica en $13,2 \%$ $\mathrm{y}$ alteraciones gastrointestinales en $16,7 \%$. El 
Tabla 1. Innovaciones terapéuticas en tuberculosis (TB)

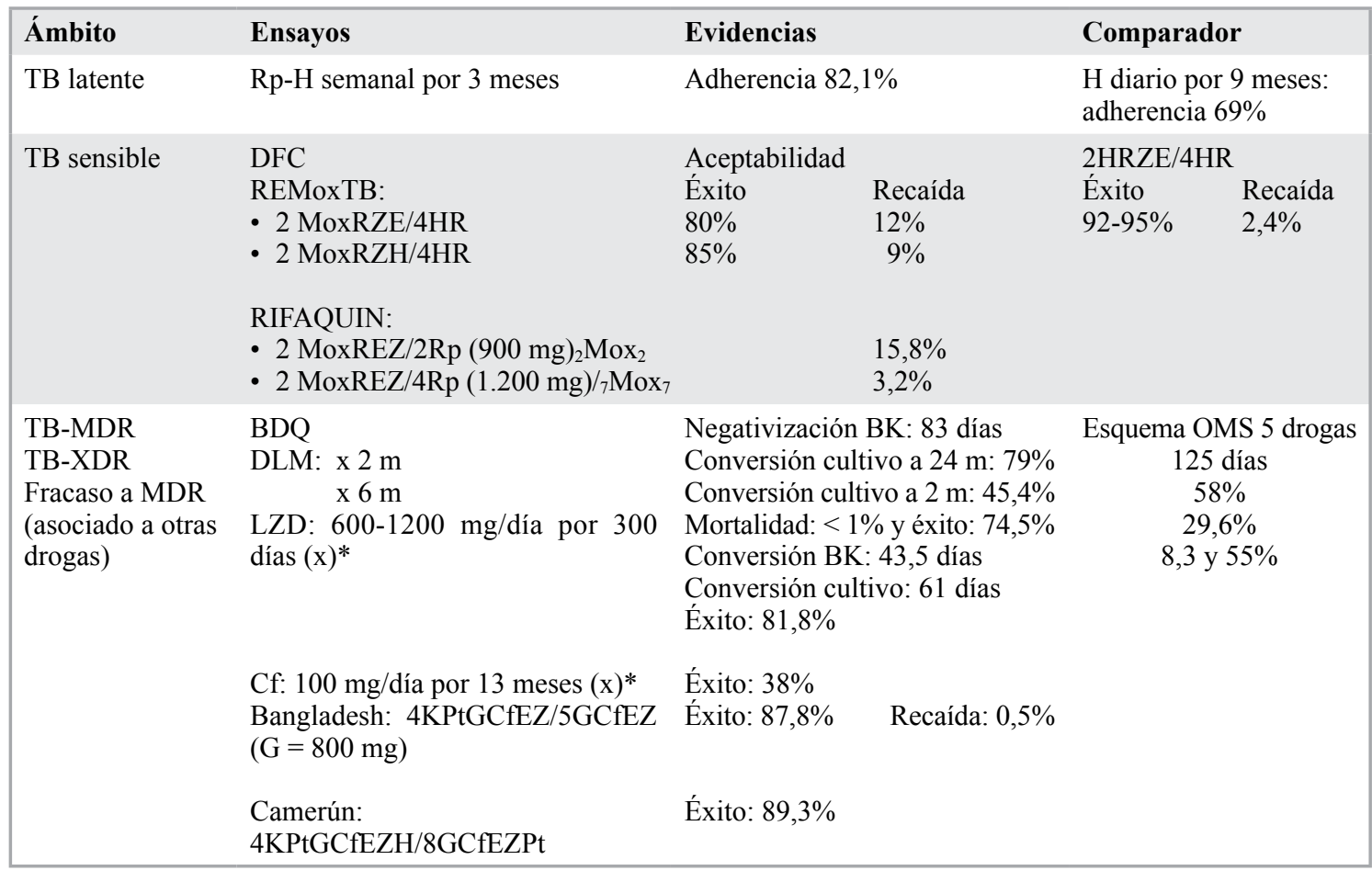

$(\mathrm{x})^{*}=$ promedio; $\mathrm{Rp}=$ rifapentina, $\mathrm{H}=$ isoniazida, $\mathrm{DFC}=$ dosis fijas combinadas, $\mathrm{R}=$ rifampicina, $\mathrm{E}=$ etambutol, $\mathrm{Z}=$ pirazinamida, Mox = moxifloxacino, $\mathrm{BDQ}=$ bedaquilina, $\mathrm{DLM}=$ delamanid, $\mathrm{LZD}=$ linezolid, $\mathrm{K}=\mathrm{kanamicina}$, $\mathrm{G}=$ gatifloxacino, $\mathrm{Cf}=$ clofazimina $. \mathrm{Pt}=$ protionamida.

LZD es eficaz pero tiene toxicidad. Nuevos protocolos deberán encontrar la dosis más adecuada y segura.

\section{Clofazimina (Cf)}

Es un agente bactericida débil que se concentra en los macrófagos y actúa uniéndose al ADN de las micobacterias, inhibiendo su crecimiento y replicación. Es un fármaco de excepción en tuberculosis, que ha sido empleado más extensamente contra la lepra. Un protocolo realizado entre los años 2008-2011 en Shangai ${ }^{22}$ en 39 pacientes sin VIH que habían fracasado con la terapia de TBMDR (la mitad eran TB-XDR), obtuvo un éxito de $38 \%$, pero un $10 \%$ abandonó la terapia y un $23 \%$ tuvo un nuevo fracaso. En este estudio se usó la dosis de $100 \mathrm{mg}$ diarios por un promedio de 13 meses en esquemas asociados a 4 o más fármacos. El tiempo de conversión del cultivo fue de 12 semanas. El 87\% presentó una reacción adversa, siendo lo más frecuente la aparición de pigmentación marrón oscura o coloración rojiza de la piel en el $79 \%$ de los casos. Las náuseas y vómitos aparecieron en el $44 \%$ y la ictiosis en $28 \%$.
En la Tabla 1 se muestra el resumen de las principales innovaciones terapéuticas en tuberculosis.

\section{Bibliografía}

1.- SMIEJA M J, MARCHETTI C A, COOK D J, SMAILL F M. Isoniazid for preventing tuberculosis in non-HIV infected persons. Cochrane database Syst rev 2000; 2: CD001363.

2.- ENA J, VALLS V. Short-course therapy with rifampin plus isoniazid for latent tuberculosis infection: a metaanalysis. Clin Infect Dis 2005; 40: 670-6.

3.- HONG KONG CHEST SERVICE/BRITISH MEDICAL RESEARCH COUNCIL. Five -year follow-up of a controlled trial of five 6-month regimens of chemotherapy for pulmonary tuberculosis. Am Rev Respir Dis 1987; 136: $1339-42$.

4.- HONG KONG CHEST SERVICE/BRITISH MEDICAL RESEARCH COUNCIL. Controlled trial of 2, 4 and 6 months of pyrazinamide in 6-month, three-times weekly regimens for smear-positive pulmonary tuberculosis, including and assessment of a combined preparation of isoniazid, rifampin and pyrazinamide: results at 30 
months. Am Rev Respir Dis 1991; 143: 700-6.

5.- TUBERCULOSIS. Informe de situación Chile 2014. Programa Nacional de Control y Eliminación de la Tuberculosis. Ministerio de Salud de Chile. Santiago, junio de 2015.

6.- FALZON D, JARAMILLO E, SCHÜNEMANN H J, ARENTZ M, BAUER M, BAYONA J, et al. WHO guidelines for the programmatic management of drugresistant tuberculosis: 2011 update. Eur Respir J 2011; 38: 516-28.

7.- $\quad$ AHUJA S, ASHKIN D, AVENDAÑO M, BANERJEE R, BAUER M, BAYONA J N, et al. Multidrug resistant pulmonary tuberculosis treatment regimens and patients outcomes: an individual patient data meta-analysis of 9,153 patients. PLoS Med 2012; 9 (8): e1001300. doi: 10.1371/journal.pmed.1001300

8.- STERLING T R, VILLARINO M E, BORISOV A S, SHANG N, GORDIN F, BLIVEN SIZEMORE E, et al. Three months of rifapentine and isoniazid for latent tuberculosis infection. N Engl J Med 2011; 365: 2155 66.

9.- GILLESPIE S H, CROOK A M, MCHUGH T D, MENDEL C M, MEREDITH S K, MURRAY S R, et al. Four-Month Moxifloxacin-based regimens for drug-sensitive tuberculosis. N Engl J Med 2014; 371: 1577-87.

10.- JINDANI A, HARRISON T, NUNN A, PHILLIPS P, CHURCHYARD G, CHARALAMBOUS S, et al. High-dose Rifapentine with moxifloxacin for pulmonary tuberculosis. N Engl J Med 2014; 371: 1599-608.

11.- DIACON A H, PYM A, GROBUSCH M P, DE LOS RÍOS J M, GOTUZZO E, VASILYEVA I, et al. Multidrug-resistant tuberculosis and culture conversión with Bedaquiline. N Engl J Med 2014; 371: 723-32.

12.- GLER M, SKRIPCONOKA V, SÁNCHEZ-GARAVITO E, XIAO H, CABRERA-RIVERO JL, VARGASVASQUEZ D, et al. Delamanid for multidrug-resistant pulmonary tuberculosis. N Engl J Med 2012; 366: 215160.

13.- SKRIPCONOKA V, DANILOVITS M, PEHME L, TOMSON T, SKENDERS G, KUMMIK T, et al. De- lamanid improves outcomes and reduces mortality for multidrug-resistant tuberculosis. Eur Respir J 2013; 41: 1393-400.

14.- VAN DEUN A, MAUG AK, SALIM MA, DAS PK, et al. Short, highly effective, and inexpensive standardized treatment of multidrug-resistant tuberculosis. Am J Respir Crit Care Med 2010; 182: 684-92. Doi:10.1164/ rccm.201001-0077OC.

15.- AUNG K J M, VAN DEUN A, DECLERCQ E, SARKER M R, DAS P K, HOSSAIN M, et al. Succesful 9 month Bangladesh regimen for multidrug-resistant tuberculosis among over 500 consecutive patients. Int J Tuberc Lung Dis 2014; 18: 1180-7.

16.- YEW W-W. High-dose fluoroquinolones in short-course regimens for treatment of MDR-TB: the way forward? Int J Tuberc Lung Dis 2013; 17: 853-4.

17.- KUABAN C, NOESKE J, RIEDER H, AIT-KHALED N, FOE A, TREBUCQ A. High effectiveness of a 12 month regimen for MDR-TB patients in Cameroon. Int J Tuberc Lung Dis 2015; 19: 517-24.

18.- CHANG K C, LEUNG C C, YEW W W, LEUNG E C, LEUNG W M, TAM C M, et al. Pyrazinamide may improve fluorquinolone-based treatment of multidrug resistant tuberculosis. Antimicrob Agents Chemother 2012; 56: 5465-75.

19.- CHANG K, YEW W W, TAM C, LEUNG C. WHO group 5 drugs and difficult multidrug-resistant tuberculosis: a systematic review with cohort analysis and meta-analysis. Antimicrobial Agents and Chemotherapy 2013; 57: 4097-104.

20.- XU H-B, JIANG R-H, LI L, XIAO H-P. Linezolid in the treatment of MDR-TB: a retrospective clinical study. Int J Tuberc Lung Dis 2012; 16: 358-63.

21.- SOTGIU G, CENTIS R, D'AMBROSIO L, ALFFENAAR J W, ANGER H A, CAMINERO J A, et al. Efficacy, safety and tolerability of linezolid containing regimens in treating MDR-TB and XDR-TB: systematic review and meta-analysis. Eur Respir J 2012; 40: 1430-42.

22.- XU HB, JIANG R-H, XIAO H-P. Clofazimine in the treatment of multidrug-resistant tuberculosis. Clin Microbiol Infect 2012; 18: 1104-10. 